\title{
Comparison of the efficacy and tolerability of gefitinib with pemetrexed maintenance after first-line platinum-based doublet chemotherapy in advanced lung adenocarcinoma: single-center experience
}

This article was published in the following Dove Press journal:

OncoTargets and Therapy

14 October 2016

Number of times this article has been viewed

\author{
Liping Lin ${ }^{1,2, *}$ \\ Juanjuan Zhao ${ }^{3, *}$ \\ Jiazhu $\mathrm{Hu}^{1,2}$ \\ Fuxi Huang ${ }^{1,2}$ \\ Jianjun $\mathrm{Han}^{1,2}$ \\ Yan $\mathrm{He}^{1,2}$ \\ Xiaolong $\mathrm{Cao}^{1,2}$ \\ 'Department of Oncology, Panyu \\ Central Hospital, ${ }^{2}$ Cancer Institute of \\ Panyu, ${ }^{3}$ School of Nursing, Sun Yat-sen \\ University, Guangzhou, People's \\ Republic of China \\ *These authors contributed equally \\ to this work
}

Correspondence: Xiaolong Cao Department of Oncology, Panyu Central Hospital, No 8 Fuyu Road East, Panyu, Guangzhou, Guangdong province, 5 I I 400, People's Republic of China Tel +862034859213

Fax +86 2034858309

Email pyzhl616@sina.com
Purpose: Both gefitinib and pemetrexed maintenance were effective therapies for advanced lung adenocarcinoma, but which is better is unclear. For patients with advanced lung adenocarcinoma, we have no idea whether we should choose gefitinib or pemetrexed maintenance in clinical practice. Here, we assessed the efficacy and tolerability of gefitinib versus pemetrexed maintenance in these patients.

Patients and methods: A total of 101 patients were identified and divided into gefitinib $(n=53)$ or pemetrexed $(n=48)$ maintenance. Epidermal growth factor receptor (EGFR) status of tumors was analyzed in 67 patients. Disease control rate (DCR), progression-free survival (PFS), and overall survival (OS) were compared between the two groups.

Results: The results showed that DCR $(79.2 \%$ vs $75 \%, P=0.642)$ was similar between gefitinib and pemetrexed groups. The PFS of gefitinib was significantly longer than that of pemetrexed ( 8 months vs 5.4 months, hazard ratio [HR]: $0.520,95 \%$ confidence interval [CI]: $0.341-0.791, P=0.002)$; however, the OS was similar (19.9 months vs 18.8 months, HR: 1.006 , 95\% CI: 0.664-1.525, $P=0.977)$. In EGFR mutation-positive patients, PFS was significantly longer in gefitinib (12 months vs 5.4 months; HR: $0.158,95 \% \mathrm{CI}: 0.074-0.333, P<0.001$ ), whereas in EGFR wild-type subgroup gefitinib had a significantly shorter PFS than that by pemetrexed (2.5 months vs 5 months; HR: 2.822 , 95\% CI: $1.137-7.005, P=0.025)$. Cox multivariate regression analysis of PFS for overall population showed that smoking status $(P=0.001)$ and maintenance regimens $(P=0.013)$ were independent prognostic factors for PFS. Both gefitinib and pemetrexed were well tolerated.

Conclusion: Gefitinib compared with pemetrexed as maintenance therapy had a significantly longer PFS and a similar OS with good tolerability in patients with advanced lung adenocarcinoma. Moreover, for EGFR mutation-positive patients, gefitinib maintenance had a significantly longer PFS; however, pemetrexed maintenance was considered more effective for EGFR wild-type patients.

Keywords: gefitinib, pemetrexed, lung adenocarcinoma, maintenance therapy

\section{Introduction}

Lung cancer is the main cause of cancer-related deaths worldwide. ${ }^{1}$ About $80 \%-85 \%$ of the cases are non-small-cell lung cancer (NSCLC), and $30 \%-40 \%$ of patients have advanced disease at the time of diagnosis. ${ }^{2}$ Epidermal growth factor receptor (EGFR) 
mutation detection in first-line setting was recommended by the National Comprehensive Cancer Network guidelines for advanced NSCLC. ${ }^{3}$ However, there were many patients whose EGFR mutation status was unknown in clinical practice due to lack of sufficient tissue, high cost of testing or limited testing technology. A national survey found that EGFR detection rate was $<20 \%$ in China, ${ }^{4}$ which meant that $\sim 80 \%$ of patients were EGFR unknown. Platinum-based doublet chemotherapy is the standard first-line therapy for these patients. ${ }^{5}$ Notwithstanding, after four to six cycles of chemotherapy, most of the patients rapidly have disease progression. Secondline therapy could prolong survival and relieve symptoms compared with placebo or best supportive care, but due to significant deterioration of performance status (PS), only $50 \%-70 \%$ of patients could get second-line chemotherapy after disease progression. ${ }^{6-8}$ Therefore, maintenance therapy, which is defined as patients who had a disease control after four to six cycles of first-line chemotherapy continue to receive therapy until disease progression or unacceptable toxicity, ${ }^{9}$ has become an important therapeutic strategy to improve the outcome.

Several meta-analyses had shown that maintenance therapy could improve PFS and OS compared with placebo, observation or best supportive care in advanced NSCLC patients. ${ }^{10-12}$ One of these studies reported that more PFS benefit could be derived in patients with adenocarcinoma than in those with squamous cell carcinoma. ${ }^{12}$ However, although the results suggested that adenocarcinoma patients should be more actively taken into account for initiating maintenance therapy, the most appropriate maintenance drug for lung adenocarcinoma is unclear. A meta-comparison of maintenance treatments by Bayesian network found that switching to or continuing pemetrexed or switching to EGFR tyrosine kinase inhibitor (TKI) for non-squamous patients showed clinically meaningful benefits of $\geq 20 \%$ reduction in hazards of death with $\geq 90 \%$ probability of outperforming no maintenance in terms of OS, ${ }^{13}$ but it could not compare the difference between the two treatments, and up to now, there are no randomized Phase III studies comparing this in lung adenocarcinoma. Moreover, these patients often had a higher EGFR-positive mutation rate; a study found that the rate of EGFR-positive mutation was $50.2 \%$ in Chinese lung adenocarcinoma patients, and if nonsmokers, it was even as high as $60.7 \% .^{14}$ Therefore, it is a difficult choice whether we should choose gefitinib or pemetrexed as maintenance therapy for advanced lung adenocarcinoma patients in clinical practice.

In this situation, we conducted this retrospective study to compare the maintenance efficacy and tolerability of gefitinib compared with pemetrexed in advanced lung adenocarcinoma. In this study, the EGFR mutation status of tumors was determined, and subgroup analyses were performed in patients who were tested for EGFR mutation status.

\section{Patients and methods}

\section{Patients}

The institutional ethics committee of Guangzhou Panyu Central Hospital approved this study. Written informed consent was obtained from each patient. Patients' inclusion criteria included 1) age $\geq 18$ years, histologically or cytologically confirmed lung adenocarcinoma; 2) stage IIIB or IV according to tumor/node/metastasis system (7th edition), ${ }^{15} 3$ ) had an objective response or stable disease (SD) after four to six cycles of first-line platinum-based chemotherapy; 4) received either gefitinib or pemetrexed maintenance therapy; and 5) had at least one measurable lesion and PS 0, 1, or 2. Blood routine, liver function, renal function, and electrocardiogram examination were routinely evaluated before maintenance therapy in all enrolled patients. Every patient had adequate hematological function, hepatic function, and renal function and was eligible for gefitinib or pemetrexed maintenance.

\section{Treatment schedule}

Included patients received either gefitinib $(250 \mathrm{mg} /$ day orally) or pemetrexed $\left(500 \mathrm{mg} / \mathrm{m}^{2}\right.$ intravenous infusion for 10 minutes on day 1 , every 3 weeks) maintenance. All pemetrexed maintenance patients received dexamethasone (4 mg orally, twice daily, on days 0-2), vitamin B12 (1 mg by intramuscular injection every 9 weeks), and folic acid ( $0.4 \mathrm{mg}$ /day orally, beginning 5-7 days before the first pemetrexed administration and continuing until 3 weeks after the last pemetrexed administration). Maintenance therapy was continued until disease progression, unacceptable adverse events or decision of the patient or physician.

\section{Clinical assessment}

Baseline evaluations including tumor and physical examinations were done before the first maintenance therapy. Computed tomography scan and/or magnetic resonance imaging was performed every 9 weeks in pemetrexed maintenance patients or 3 months in gefitinib maintenance patients to confirm the response on maintenance phase. After treatment discontinuation, the follow-up interval was every 3 months. Tumor response was evaluated using Response Evaluation Criteria in Solid Tumors 1.1 for measurable disease in combination with assessments of nonmeasurable disease, ${ }^{16}$ and the response was confirmed after 4 weeks. Before every 
cycle therapy, toxicities were assessed by adverse events and laboratory results according to the National Cancer Institute Common Terminology Criteria, version 4.0.

\section{EGFR mutation detection}

Tumor EGFR mutation status was assessed by analysis of DNA extracted from formalin-fixed paraffin-embedded (FFPE) archival tumor tissues at the Pathology Department of Panyu Central Hospital. DNA was extracted by the FFPE sample DNA isolation kit (centrifugal column type) (AmoyDx ${ }^{\circledR}$, Xiamen, China) according to the protocol described in the manufacturer's instructions. EGFR 29 Mutations Detection Kit (AmoyDx) (including the most common 29 EGFR mutations and classified into 19 deletions, T790M, L858R, L861Q, G719X, S768I, and Insertion assays) was applied to detect EGFR mutations by real-time polymerase chain reaction following the user manual. Cycling parameters from the user manual were carried out, and the fluorescent signal was collected from FAM and HEX channels. The final results were reported according to the signal curve.

\section{Statistical analysis}

The clinical characteristics between the two groups were evaluated using the Fisher's exact test. Reverse KaplanMeier estimate was used to calculate median follow-up. PFS was calculated from the date of maintenance therapy to disease progression or death, whichever came first. OS was calculated from the date of maintenance therapy to the date of death or last clinical follow-up. PFS and OS were estimated from the date of maintenance therapy using the Kaplan-Meier method, and the results for each treatment arm were compared using the log-rank test. Cox regression model with treatment groups as the only cofactor was performed on PFS and OS to compare the two treatment strategies over the entire study (EGFR mutation-positive subgroup, EGFR wild-type subgroup and EGFR unknown subgroup). A Cox multivariate regression analysis was done on PFS for the overall population, and covariates included sex, PS, smoking status, maintenance regimens and response of first-line chemotherapy. Statistical significance was set at 5\%. All tests were two-sided, and analyses were carried out with SPSS software, version 17.0 (SPSS Inc., Chicago, IL, USA).

\section{Results}

\section{Patients' characteristics}

From September 1, 2009, to December 30, 2013, there were 328 advanced NSCLC patients who had disease control after first-line platinum-based doublet chemotherapy in the Department of Oncology of Guangzhou Panyu Central Hospital. A total of 225 of 328 patients had received maintenance therapy, and 158 of these 225 patients had adenocarcinoma. We selected 101 of 158 patients for our study. The other 57 patients were excluded because of three reasons: 1) 31 patients received gemcitabine maintenance, 2) 15 patients received docetaxel maintenance, and 3) 11 patients received erlotinib maintenance.

A total of 101 consecutive treated patients from September 2009 to December 2013 were identified. Among these patients (median age: 57 years; range: $35-78$ years), 54 $(53.5 \%)$ were never-smokers and $36(35.6 \%)$ were females. In total, $29(28.7 \%)$ patients had received pemetrexedplatinum first-line chemotherapy, 24 (23.8\%) patients had received taxane-platinum first-line chemotherapy and 48 $(47.5 \%)$ had received gemcitabine-platinum first-line chemotherapy. In first-line chemotherapy, there were 52 (51.5\%) patients showing partial response (PR) and 49 (48.5\%) patients showing SD. A total of 101 patients were divided into gefitinib group $(n=53)$ or pemetrexed group $(n=48)$. The proportion of female patients was significantly higher in gefitinib group ( $45.3 \%$ vs $25 \%, P=0.039)$; the other demographic and clinical characteristics were balanced between the two groups (Table 1). The median patient follow-up was 25 months (range: 18-44.2 months).

\section{EGFR detection results}

In 101 lung adenocarcinoma patients, we had tested EGFR mutation status in 67 patients, whereas the remaining 34 patients had been not tested because of insufficient tissue samples. Among the tested patients, 41 of 67 (61.2\%) were EGFR mutation positive (28 [68.3\%] exon 19 deletions, 12 [29.3\%] L858R, 1 [2.4\%] L861Q) and 26 of 67 (38.8\%) were EGFR wild-type (Table 1).

\section{Tumor response}

Median duration of gefitinib maintenance was 8 (range: 1-30) months, and the median number of pemetrexed maintenance cycles delivered was 4 (range: 1-16). Among all 101 patients, there were no complete response, 27 (26.7\%) patients achieved PR, 51 (50.5\%) patients had $\mathrm{SD}$, and $23(22.8 \%)$ patients had progressive disease (PD). In gefitinib group, 17 (32.1\%) patients had PR, 25 (47.2\%) patients had SD, and $11(20.8 \%)$ patients had PD, whereas in pemetrexed group, 10 (20.8\%), 26 (54.2\%), and 12 (25\%) patients had PR, SD, and PD, respectively. Gefitinib group had a mildly higher proportion of patients showing response in overall population, and the disease control rates 
Table I Patients' clinical characteristics and EGFR mutation status

\begin{tabular}{|c|c|c|c|}
\hline Characteristics & $\begin{array}{l}\text { Gefitinib group } \\
(n=53)(\%)\end{array}$ & $\begin{array}{l}\text { Pemetrexed group } \\
(n=48)(\%)\end{array}$ & $P$-value \\
\hline Median age (range), years & $59(35-77)$ & $57(36-78)$ & 0.626 \\
\hline Age, years & & & 1.000 \\
\hline$<65$ & $38(71.7)$ & $35(72.9)$ & \\
\hline$\geq 65$ & I5 (28.3) & $13(27.1)$ & \\
\hline Gender & & & 0.039 \\
\hline Male & $29(54.7)$ & $36(75.0)$ & \\
\hline Female & $24(45.3)$ & $12(25.0)$ & \\
\hline Smoking status & & & 0.166 \\
\hline Smoker & $21(39.6)$ & $26(54.2)$ & \\
\hline Never-smoker & $32(60.4)$ & $22(45.8)$ & \\
\hline Stage & & & 0.496 \\
\hline IIIB & $2(3.8)$ & $0(0.0)$ & \\
\hline IV & $51(96.2)$ & $48(100.0)$ & \\
\hline Performance status & & & 1.000 \\
\hline $0-1$ & $48(90.6)$ & 44 (91.7) & \\
\hline 2 & $5(9.4)$ & $4(8.3)$ & \\
\hline First-line chemotherapy regimen & & & 0.051 \\
\hline Pemetrexed-platinum & $10(18.9)$ & $19(39.6)$ & \\
\hline Docetaxel-platinum & $16(30.2)$ & $8(16.7)$ & \\
\hline Gemcitabine-platinum & $27(50.9)$ & $21(43.8)$ & \\
\hline Response of first-line chemotherapy & & & 0.691 \\
\hline Partial response & $26(49.1)$ & $26(54.2)$ & \\
\hline Stable disease & $27(50.9)$ & $22(45.8)$ & \\
\hline EGFR status & & & 0.743 \\
\hline EGFR mutation-positive & $23(43.4)$ & $18(37.5)$ & \\
\hline Exon $19 \mathrm{del}$ & $18(34.0)$ & $10(20.8)$ & \\
\hline L858R & $4(7.5)$ & $8(16.7)$ & \\
\hline L86IQ & $\mathrm{I}(1.9)$ & $0(0.00)$ & \\
\hline EGFR wild-type & $12(22.6)$ & $14(29.2)$ & \\
\hline EGFR mutation unknown & $18(34.0)$ & $16(33.3)$ & \\
\hline
\end{tabular}

Abbreviation: EGFR, epidermal growth factor receptor.

were similar in gefitinib compared with pemetrexed group regardless of overall population ( $79.2 \%$ vs $75 \%, P=0.642)$, EGFR mutation-positive population $(100 \%$ vs $88.9 \%$, $P=0.187)$, EGFR wild-type population ( $50 \%$ vs $64.3 \%$, $P=0.692)$, and EGFR unknown population $(72.2 \%$ vs $68.8 \%, P=1.000$ ) (Table 2).

\section{PFS and Cox multivariate regression analysis}

The median PFS of gefitinib was longer than that of pemetrexed (8 [95\% CI: 5.5-10.5] months vs 5.4 [95\% CI: 4.3-6.5] months; HR: $0.520,95 \%$ CI: 0.341-0.791, $P=0.002$ ) (Table 2 and Figure 1A). In EGFR mutation-positive subgroup, PFS was also significantly longer in gefitinib group than pemetrexed group (12 [95\% CI: $10.5-13.5]$ months vs 5.4 [95\% CI: 2.9-7.9] months; HR: $0.158,95 \%$ CI: $0.074-0.333$, $P<0.001$ ) (Table 2 and Figure 1B), whereas, in EGFR wild-type subgroup, gefitinib had a significantly shorter PFS than pemetrexed (2.5 [95\% CI: $0.8-4.2]$ months vs 5
[95\% CI: 3.7-6.3] months; HR: 2.822, 95\% CI: 1.137-7.005, $P=0.025$ ) (Table 2 and Figure 1C); in EGFR unknown subgroup, PFS was similar between gefitinib and pemetrexed groups (6 [95\% CI: 4.6-7.4] months vs 5.6 [95\% CI: 4.8-6.4] months; HR: $0.850,95 \%$ CI: 0.426-1.699, $P=0.646$ ) (Table 2 and Figure 1D).

The results of Cox multivariate regression analysis of PFS for overall population showed that smoking status and maintenance regimens were independent prognostic factors for PFS, never-smokers $(P=0.001)$ and gefitinib maintenance $(P=0.013)$, suggesting a longer PFS for lung adenocarcinoma patients (Table 3 ).

\section{OS}

The median OS was not significantly different between gefitinib and pemetrexed groups regardless of population: whole population (19.9 [95\% CI: 17.4-22.4] months vs 18.8 [95\% CI: 17.7-19.9] months; HR: 1.006, 95\% CI: 0.664-1.525, $P=0.977$ ) (Table 2 and Figure 2A); EGFR mutation-positive 


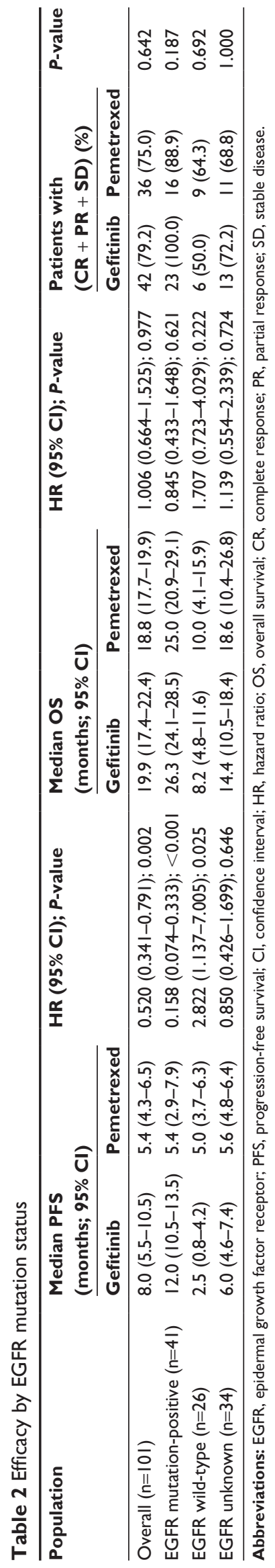

subgroup (26.3 [95\% CI: 24.1-28.5] months vs 25 [95\% CI: 20.9-29.1] months; HR 0.845, 95\% CI: $0.433-$ 1.648, $P=0.621$ ) (Table 2 and Figure 2B); EGFR wild-type subgroup (8.2 [95\% CI: 4.8-11.6] months vs 10 [95\% CI: 4.1-15.9] months; HR: 1.707, 95\% CI: 0.723-4.029, $P=0.222$ ) (Table 2 and Figure 2C) or EGFR unknown subgroup (14.4 [95\% CI: $10.5-18.4]$ months vs 18.6 [95\% CI: 10.4-26.8] months; HR: 1.139, 95\% CI: 0.554-2.339, $P=0.724$ ) (Table 2 and Figure 2D).

\section{Second- and third-line therapy}

The proportions of patients receiving second-line ( $71.7 \% \mathrm{vs}$ $77.1 \%, P=0.650)$ and third-line ( $32.1 \%$ vs $45.8 \%, P=0.219$ ) treatments were similar between gefitinib and pemetrexed groups. Docetaxel was the most common second-line therapy in gefitinib group, while gefitinib was the most common in pemetrexed group as salvage treatments. Docetaxel was also the most common third-line chemotherapy in gefitinib group and it was gefitinib in pemetrexed group (Table 4).

\section{Toxicities}

Table 5 lists the most common adverse events (AEs). In gefitinib group, rash was the most frequent $\mathrm{AE}$, with $15.1 \%$ of patients experiencing grade 3-4 rash; moreover, there was one $(1.9 \%)$ patient who experienced grade 3 interstitial lung disease. In pemetrexed group, hematologic toxicities such as leukopenia (4.2\%), neutropenia (2.1\%), and anemia (2.1\%) were the most common grade 3-4 AEs. Nausea, fatigue, and diarrhea were the common non-hematologic toxicities in the two groups.

\section{Discussion}

Both gefitinib and pemetrexed are currently optional as maintenance therapy for advanced lung adenocarcinoma patients. Our investigation assessed the maintenance efficacy and tolerability of gefitinib compared with pemetrexed in these patients. The results showed that gefitinib had a mildly higher proportion of patients showing PR. The median PFS was significantly longer for patients in gefitinib than pemetrexed, and Cox multivariate regression analysis showed that smoking status and maintenance regimen were independent prognostic factors; however, the benefit of PFS could not translate into OS benefit. Furthermore, in subgroup analysis, there was a similar benefit in PFS derived from gefitinib in EGFR mutation-positive subgroup as the result of overall population; however, it was pemetrexed obtaining significantly longer PFS in EGFR wild-type subgroup. Moreover, there were some differences in toxicities between the two treatments, but all were manageable. 

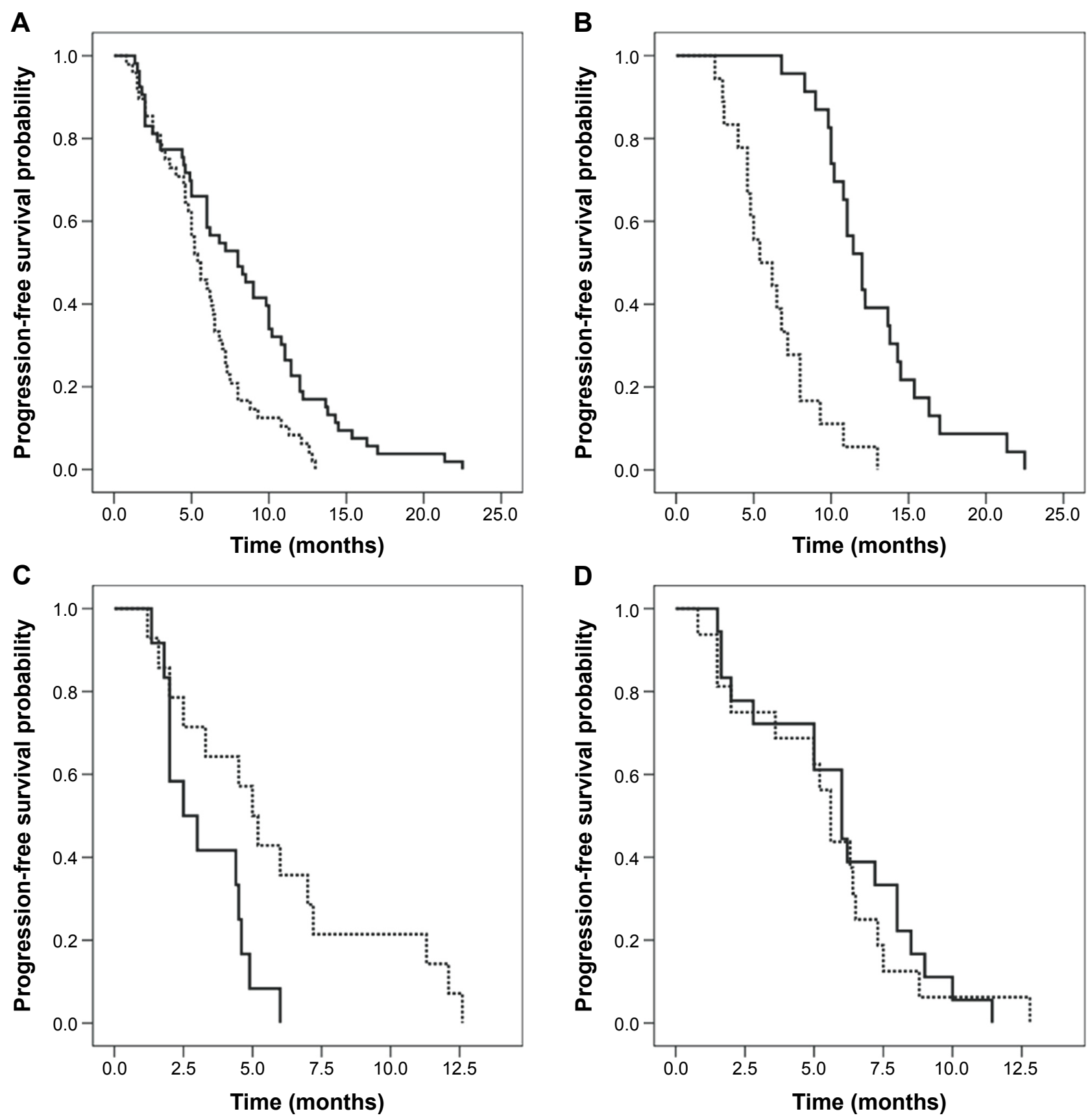

\section{Group \\ $\neg$ Gefitinib ...: Pemetrexed}

Figure I Kaplan-Meier curve for PFS: (A) overall population, (B) EGFR mutation-positive population, (C) EGFR wild-type population, and (D) EGFR unknown population. Abbreviations: PFS, progression-free survival; EGFR, epidermal growth factor receptor.

Table 3 Cox multivariate regression analysis of PFS for overall population

\begin{tabular}{lllll}
\hline Variable & Good covariates & HR & $\mathbf{9 5 \%} \mathbf{C l}$ & $\mathbf{P}$-value \\
\hline Smoking status & Never-smoker & 0.491 & $0.319-0.755$ & 0.001 \\
Maintenance & Gefitinib & 0.583 & $0.38 I-0.892$ & 0.013 \\
regimens & maintenance & & & \\
\hline
\end{tabular}

Abbreviations: PFS, progression-free survival; $\mathrm{HR}$, hazard ratio; $\mathrm{Cl}$, confidence interval.
Two clinical trials respectively compared gefitinib with pemetrexed as maintenance therapy in advanced lung adenocarcinoma or never-smoker NSCLC. A recent Chinese randomized study reported that gefitinib had a significantly higher DCR than pemetrexed in maintenance therapy in advanced lung adenocarcinoma, but the author did not report the survival results. ${ }^{17}$ In contrast with the results of 
A

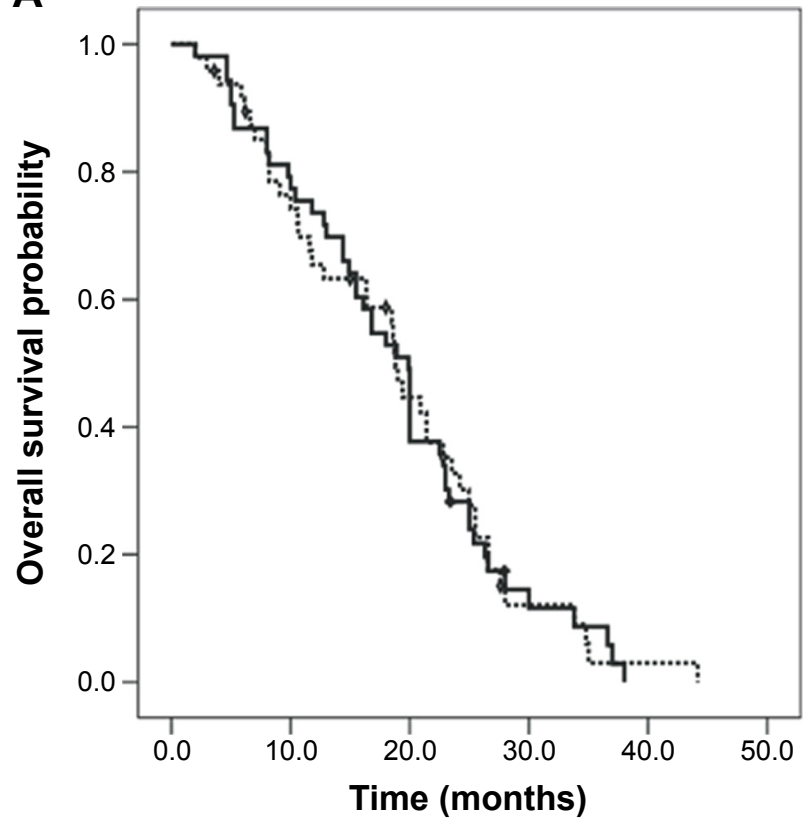

C

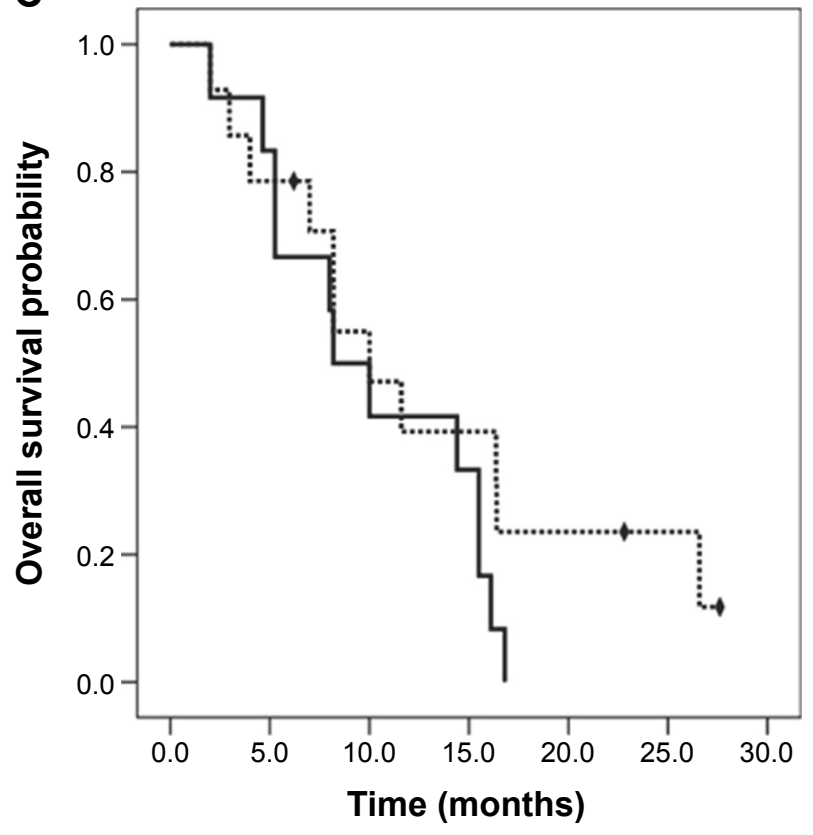

B

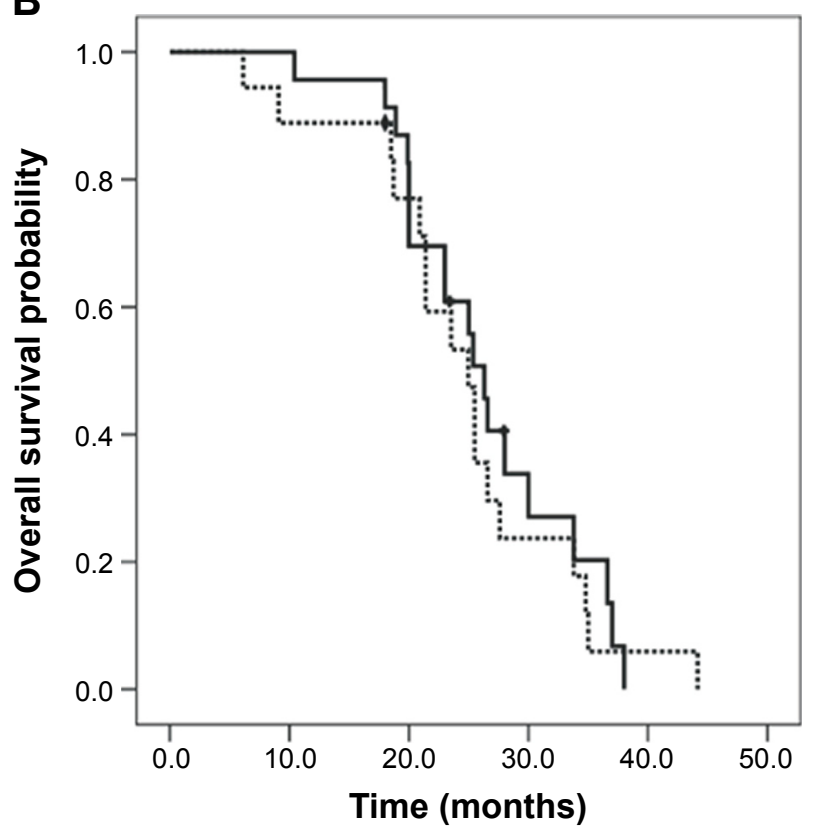

D

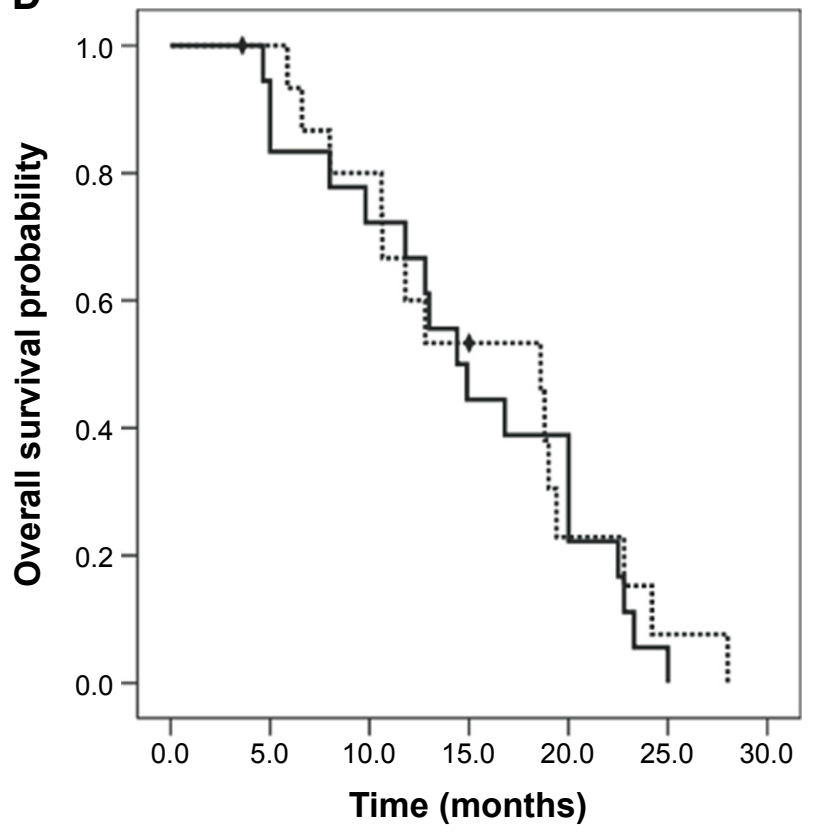

Group

$\neg$ Gefitinib ...: Pemetrexed + Gefitinib-censored $\diamond$ Pemetrexed-censored

Figure 2 Kaplan-Meier curve for OS: (A) overall population, (B) EGFR mutation-positive population, (C) EGFR wild-type population, and (D) EGFR unknown population. Abbreviations: OS, overall survival; EGFR, epidermal growth factor receptor.

this study, the DCR between gefitinib and pemetrexed in our research was not significantly different, which was similar to the results of a randomized Phase II clinical trial initiated by Ahn et al, which investigated the efficacy after first-line treatment with pemetrexed-cisplatin followed sequentially by gefitinib $(\mathrm{PC} / \mathrm{G})$ or pemetrexed $(\mathrm{PC} / \mathrm{P})$ in East Asian never-smoker advanced NSCLC patients (all patients were
EGFR unknown and $84.3 \%$ patients had non-squamous carcinoma). ${ }^{18}$ The results showed that the response rate was similar in both arms. Moreover, it was notable that PFS (calculated from first-line treatment) was numerically longer in $\mathrm{PC} / \mathrm{G}, \mathrm{OS}$ (calculated from first-line treatment) was numerically higher in $\mathrm{PC} / \mathrm{P}$, and similar trend was shown in nonsquamous carcinoma. Similar to this, we had also observed 
Table 4 Second- and third-line chemotherapy

\begin{tabular}{lll}
\hline $\begin{array}{l}\text { Chemotherapy } \\
\text { regimens }\end{array}$ & $\begin{array}{l}\text { Gefitinib group } \\
(\mathbf{n}=\mathbf{5 3}) \mathbf{( \% )}\end{array}$ & $\begin{array}{l}\text { Pemetrexed group } \\
(\mathbf{n}=\mathbf{4 8}) \mathbf{( \% )}\end{array}$ \\
\hline Second-line chemotherapy & $38(71.7)$ & $37(77.1)$ \\
Docetaxel & $14(26.4)$ & $13(27.1)$ \\
Docetaxel-platinum & $0(0.0)$ & $1(2.1)$ \\
Erlotinib & $0(0.0)$ & $4(8.3)$ \\
Gefitinib & $0(0.0)$ & $17(35.4)$ \\
Gemcitabine & $8(15.1)$ & $1(2.1)$ \\
Gemcitabine-platinum & $2(3.8)$ & $0(0.0)$ \\
Pemetrexed & $13(24.5)$ & $0(0.0)$ \\
Irinotecan & $I(1.9)$ & $0(0.0)$ \\
Irinotecan-platinum & $0(0.0)$ & $1(2.1)$ \\
Third-line chemotherapy & $17(32.1)$ & $22(45.8)$ \\
Vinorelbine & $I(1.9)$ & $0(0.0)$ \\
Docetaxel & $8(15.1)$ & $6(12.5)$ \\
Gefitinib & $0(0.0)$ & $8(16.7)$ \\
Gemcitabine & $2(3.8)$ & $3(6.2)$ \\
Pemetrexed & $3(5.7)$ & $1(2.1)$ \\
Pemetrexed-platinum & $0(0.0)$ & $\mathrm{I}(2.1)$ \\
S-I & $2(3.8)$ & $\mathrm{I}(2.1)$ \\
Irinotecan & $\mathrm{I}(1.9)$ & $\mathrm{I}(2.1)$ \\
Paclitaxel-platinum & $0(0.0)$ & $\mathrm{I}(2.1)$ \\
\hline
\end{tabular}

a benefit of PFS in gefitinib group; however, probably due to crossover, the advantage of PFS on gefitinib group could not translate to the benefit of OS.

Compared with pemetrexed single agent, gefitinib was the favored selection in second-line treatment for EGFR mutation-positive patients $;{ }^{19}$ in contrast, it was pemetrexed for EGFR wild-type patients in advanced NSCLC. ${ }^{20,21}$ However, there were no trials that had compared the maintenance efficacy between gefitinib and pemetrexed in advanced lung adenocarcinoma according to different EGFR mutation statuses. Therefore, whether the conclusions from second-line treatment could be extended to maintenance therapy remains unclear. The subgroup analysis of INFORM and SATURN study had proven that gefitinib and erlotinib had longer PFS and OS than placebo for EGFR mutation-positive patients and similar PFS and OS versus placebo for EGFR wild-type patients in maintenance therapy. ${ }^{8,22}$ These findings suggest that the exact EGFR mutation status should be considered in maintenance treatment to guide the precision of therapy. In this study, a significantly longer PFS was observed in gefitinib than pemetrexed among EGFR mutation-positive patients. In contrast, it was pemetrexed that had a significantly longer PFS in EGFR wild-type subgroup. However, the OS was similar in each subgroup, which was probably due to crossover of subsequent treatments. The results of PFS in our study were in accordance with the conclusion of studies comparing pemetrexed and gefitinib in second-line therapy for EGFR mutation-positive or wild-type NSCLC. So, based on our retrospective results, we first reported that the efficacy of gefitinib was better than pemetrexed for EGFR mutationpositive patients, and pemetrexed had more survival benefit for EGFR wild-type patients than gefitinib on maintenance phase. EGFR mutation status was the strongest predictive factor for maintenance efficacy of gefitinib and pemetrexed.

Although skin rash and diarrhea were the most common AEs with gefitinib, most patients' AEs were in grade 1/2. It was worthy to note that one patient experienced grade 3 interstitial lung disease in gefitinib. It might be due to the old age of this patient and a long history of smoking and radioactive pulmonary fibrosis previously. Some studies

Table 5 Drug-related toxicities

\begin{tabular}{|c|c|c|c|c|}
\hline \multirow[t]{2}{*}{ Toxicities } & \multicolumn{2}{|c|}{ Gefitinib group $(n=53)(\%)$} & \multicolumn{2}{|c|}{ Pemetrexed group $(n=48)(\%)$} \\
\hline & All grades & $\geq$ Grade 3 & All grades & $\geq$ Grade 3 \\
\hline \multicolumn{5}{|l|}{ Hematological toxicities } \\
\hline Leukopenia & $5(9.4)$ & $0(0.0)$ & $9(18.7)$ & $2(4.2)$ \\
\hline Neutropenia & $2(3.8)$ & $0(0.0)$ & $7(14.6)$ & $\mathrm{I}(2 . \mathrm{I})$ \\
\hline Thrombocytopenia & $0(0.0)$ & $0(0.0)$ & I (2.I) & $0(0.0)$ \\
\hline Anemia & $2(3.8)$ & $0(0.0)$ & $6(12.5)$ & $\mathrm{I}(2.1)$ \\
\hline \multicolumn{5}{|l|}{ Non-hematological toxicities } \\
\hline Interstitial lung disease & I (I.9) & I (I.9) & $0(0.0)$ & $0(0.0)$ \\
\hline Alanine aminotransferase & $6(11.3)$ & $0(0.0)$ & $3(6.2)$ & $0(0.0)$ \\
\hline Aspartate aminotransferase & $4(7.5)$ & $0(0.0)$ & $2(4.2)$ & $0(0.0)$ \\
\hline Nausea & $2(3.8)$ & $0(0.0)$ & $8(16.7)$ & $0(0.0)$ \\
\hline Vomiting & $0(0.0)$ & $0(0.0)$ & $4(8.3)$ & $0(0.0)$ \\
\hline Fatigue & $5(9.4)$ & $0(0.0)$ & $8(16.7)$ & $\mathrm{I}(2 . \mathrm{I})$ \\
\hline Diarrhea* & $12(22.6)$ & $0(0.0)$ & $0(0.0)$ & $0(0.0)$ \\
\hline Rash* & $20(37.7)$ & $8(15.1)$ & $0(0.0)$ & $0(0.0)$ \\
\hline Anorexia & $5(9.4)$ & $0(0.0)$ & $6(12.5)$ & I (2.I) \\
\hline
\end{tabular}

Notes: $* P<0.05$ for the rates of diarrhea and rash between two groups. 
reported that the risk of interstitial lung disease in such patients was significantly higher than others during EGFRTKI treatment. ${ }^{23,24}$ As expected, grade 3/4 toxicities were also very low in pemetrexed and only slightly higher in hematologic toxicities such as leukopenia, neutropenia, and anemia, and there were no unexpected toxicities. These results indicate that the toxicities of the two treatments were somewhat different, but were all well tolerated, which was similar to the previous findings. ${ }^{8,25,26}$ Either treatment could be used in maintenance after induction chemotherapy without increased toxicity.

Of course, there were some limitations in our study. 1) It was a small sample size retrospective analysis conducted at a single center, which reduced the reliability and statistical power of comparison between the two treatment arms. 2) The time span of our study was almost 4 years, which was too long. As we know, the treatment methods of advanced NSCLC had been changed greatly in these years; there might be many hybrid factors during and after maintenance phase, which might influence the actual treatment effect. 3) This study enrolled the patients in our daily clinical practice, which could have biased the results such as drug-related toxicities graded and recorded. 4) On maintenance phase, the response evaluation time interval was different, which was 3 months in gefitinib and 9 weeks in pemetrexed, which was likely to bias the PFS assessment.

\section{Conclusion}

Our study shows that maintenance therapy with gefitinib compared with pemetrexed had a significantly longer PFS and a similar OS. For EGFR mutation-positive patients, gefitinib significantly prolonged PFS versus pemetrexed, but compared with gefitinib, it was pemetrexed that had a significantly longer PFS for EGFR wild-type patients. In the future, we look forward to design a prospective randomized controlled study to compare the efficacy of these two treatments in advanced lung adenocarcinoma to optimize maintenance strategy.

\section{Acknowledgments}

This work was supported by Guangzhou Medicines \& Health Science and Technology Project (No 20141A010102).

\section{Disclosure}

The authors report no conflicts of interest in this work.

\section{References}

1. Siegel RL, Miller KD, Jemal A. Cancer statistics, 2015. CA Cancer J Clin. 2015;65(1):5-29.
2. Mountain CF. Revisions in the International System for Staging Lung Cancer. Chest. 1997;111(6):1710-1717.

3. National Comprehensive Cancer Network. NCCN Clinical Practice Guidelines in Oncology: Non-Small Cell Lung Cancer, Version 4; 2016. Available from: http://www.nccn.org/professionals/physician_gls/pdf/ nscl.pdf. Accessed January 19, 2016.

4. Xue $\mathrm{C}, \mathrm{Hu} Z$, Jiang W, et al. National survey of the medical treatment status for non-small cell lung cancer (NSCLC) in China. Lung Cancer. 2012;77(2):371-375.

5. Reck M, Popat S, Reinmuth N, et al. Metastatic non-small-cell lung cancer (NSCLC): ESMO Clinical Practice Guidelines for diagnosis, treatment and follow-up. Ann Oncol. 2014;25(suppl 3):iii27-iii39.

6. Tassinari D, Scarpi E, Sartori S, et al. Second-line treatments in non-small cell lung cancer. A systematic review of literature and meta-analysis of randomized clinical trials. Chest. 2009;135(6):1596-1609.

7. Ciuleanu T, Brodowicz T, Zielinski C, et al. Maintenance pemetrexed plus best supportive care versus placebo plus best supportive care for non-small-cell lung cancer: a randomised, double-blind, phase 3 study. Lancet. 2009;374(9699):1432-1440.

8. Zhang L, Ma S, Song X, et al. Gefitinib versus placebo as maintenance therapy in patients with locally advanced or metastatic non-small-cell lung cancer (INFORM; C-TONG 0804): a multicentre, double-blind randomised phase 3 trial. Lancet Oncol. 2012;13(5):466-475.

9. Stinchcombe TE, Socinski MA. Maintenance therapy in advanced nonsmall cell lung cancer: current status and future implications. $J$ Thorac Oncol. 2011;6(1):174-182.

10. Zhang C, Huang C, Wang J, et al. Maintenance or consolidation therapy for non-small-cell lung cancer: a meta-analysis involving 5841 subjects. Clin Lung Cancer. 2015; 16:e15-e23.

11. Lu S, Yu Y, Chen Z, Ye X, Li Z, Niu X. Maintenance therapy improves survival outcomes in patients with advanced non-small cell lung cancer: a meta-analysis of 14 studies. Lung. 2015;193:805-814.

12. Zhou F, Jiang T, Ma W, Gao G, Chen X, Zhou C. The impact of clinical characteristics on outcomes from maintenance therapy in non-small cell lung cancer: asystematic review with meta-analysis. Lung Cancer. 2015;89(2):203-211.

13. Tan PS, Lopes G, Acharyya S, Bilger M, Haaland B. Bayesian network meta-comparison of maintenance treatments for stage IIIb/IV non-small-cell lung cancer (NSCLC) patients with good performance status not progressing after first-line induction chemotherapy: results by performance status, EGFR mutation, histology and response to previous induction. Eur J Cancer. 2015;51:2330-2344.

14. Shi Y, Li J, Zhang S, et al. Molecular epidemiology of EGFR mutations in Asian patients with advanced non-small-cell lung cancer of adenocarcinoma histology - Mainland China Subset Analysis of the PIONEER study. PLoS One. 2015;10(11):e0143515.

15. Goldstraw P, Crowley J, Chansky K, et al. The IASLC Lung Cancer Staging Project: proposals for the revision of the TNM stage groupings in the forthcoming (seventh) edition of the TNM classification of malignant tumours. J Thorac Oncol. 2007;2(8):706-714.

16. Eisenhauer EA, Therasse P, Bogaerts J, et al. New response evaluation criteria in solid tumours: revised RECIST guideline (version 1.1). Eur J Cancer. 2009;45(2):228-247.

17. Xu YH, Mei JS, Zhou J. Randomized study of gefitinib versus pemetrexed as maintenance treatment in patients with advanced glandular non-small cell lung cancer. Int J Clin Exp Med. 2015;8(4):6242-6246.

18. Ahn MJ, Yang JC, Liang J, et al. Randomized phase II trial of first-line treatment with pemetrexed-cisplatin, followed sequentially by gefitinib or pemetrexed, in East Asian, never-smoker patients with advanced non-small cell lung cancer. Lung Cancer. 2012;77(2):346-352.

19. Sun JM, Lee KH, Kim SW, et al. Gefitinib versus pemetrexed as second-line treatment in patients with nonsmall cell lung cancer previously treated with platinum-based chemotherapy (KCSG-LU08-01): an open-label, phase 3 trial. Cancer. 2012;118(24):6234-6242.

20. Kim YS, Cho EK, Woo HS, et al. Randomized phase II study of pemetrexed versus gefitinib in previously treated patients with advanced non-small cell lung cancer. Cancer Res Treat. 2016;48(1):80-87. 
21. Zhou Q, Cheng Y, Yang JJ, et al. Pemetrexed versus gefitinib as a second-line treatment in advanced nonsquamous nonsmall-cell lung cancer patients harboring wild-type EGFR (CTONG0806): a multicenter randomized trial. Ann Oncol. 2014;25(12):2385-2391.

22. Cappuzzo F, Ciuleanu T, Stelmakh L, et al. Erlotinib as maintenance treatment in advanced non-small-cell lung cancer: a multicentre, randomised, placebo-controlled phase 3 study. Lancet Oncol. 2010;11(6): 521-529.

23. Kuo LC, Lin PC, Wang KF, Yuan MK, Chang SC. Successful treatment of gefitinib-induced acute interstitial pneumonitis with high-dose corticosteroid: a case report and literature review. Med Oncol. 2011; 28(1):79-82.

24. Johkoh T, Sakai F, Kusumoto M, et al. Association between baseline pulmonary status and interstitial lung disease in patients with nonsmall-cell lung cancer treated with erlotinib-a cohort study. Clin Lung Cancer. 2014;15(6):448-454.
25. Paz-Ares LG, de Marinis F, Dediu M, et al. PARAMOUNT: final overall survival results of the phase III study of maintenance pemetrexed versus placebo immediately after induction treatment with pemetrexed plus cisplatin for advanced nonsquamous non-small-cell lung cancer. J Clin Oncol. 2013;31(23):2895-2902.

26. Pujol JL, Paz-Ares L, de Marinis F, et al. Long-term and low-grade safety results of a phase III study (PARAMOUNT): maintenance pemetrexed plus best supportive care versus placebo plus best supportive care immediately after induction treatment with pemetrexed plus cisplatin for advanced nonsquamous non-small-cell lung cancer. Clin Lung Cancer. 2014;15(6):418-425.

\section{Publish your work in this journal}

OncoTargets and Therapy is an international, peer-reviewed, open access journal focusing on the pathological basis of all cancers, potential targets for therapy and treatment protocols employed to improve the management of cancer patients. The journal also focuses on the impact of management programs and new therapeutic agents and protocols on

\section{Dovepress}

patient perspectives such as quality of life, adherence and satisfaction. The manuscript management system is completely online and includes a very quick and fair peer-review system, which is all easy to use. Visit http://www.dovepress.com/testimonials.php to read real quotes from published authors. 